\title{
Combined test of serum CgA and NSE improved the power of prognosis prediction of NF-pNETs
}

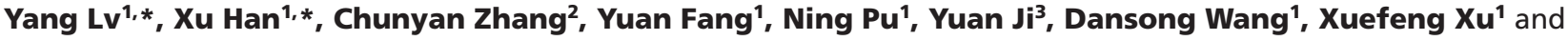 \\ Wenhui Lou' \\ ${ }^{1}$ Department of General Surgery, Zhongshan Hospital, Fudan University, Shanghai, China \\ 2Department of Clinical Laboratory, Zhongshan Hospital, Fudan University, Shanghai, China \\ ${ }^{3}$ Department of Pathology, Zhongshan Hospital, Fudan University, Shanghai, China \\ Correspondence should be addressed to W Lou or X Xu: lou.wenhui@zs-hospital.sh.cn or xuefengxu87@aliyun.com \\ *(Y Lv and $\mathrm{X}$ Han contributed equally to this work)
}

\begin{abstract}
Purpose: Chromogranin A ( $\mathrm{CgA}$ ) and neuron-specific enolase (NSE) are important markers for neuroendocrine tumors; however, the clinical value of combining these markers has not been well studied. In this study, we investigated the utility of each marker individually and in combination for patients with nonfunctional pancreatic neuroendocrine tumors (NF-pNETs).

Patients and Methods: In this study, NF-pNET patients and controls were recruited from December 2011 to March 2016; 784 serum samples from peripheral vein were collected. The clinical characteristics and biomarker values of all the individuals were recorded and analyzed. Tumor burdens were calculated by CT/MRI scan. Receiver-operating characteristic curves were constructed to assess the diagnostic predictive values; sensitivity and specificity were calculated to determine the cut-off value. Therapeutic responses reflected on the changes of the biomarkers' concentration were assessed by the RECIST criterion. Clinical relations between the prognosis and the biomarker values were also analyzed. Statistical significance was defined as $P$ value less than 0.05 . Results: Among the 167 NF-pNETs patients, 82 were males $(49.1 \%)$ and the mean age was 50.0 (17.4). The mean CgA values of G1, G2 and G3 NF-pNENs were 75, 121 and $134 \mu \mathrm{g} / \mathrm{L}(P<0.05)$, respectively. In NF-pNETs, CgA correlated with the WHO tumor grade (WHO G1 vs G2, $P<0.05$ ); the linear regression relationships were found between the tumor burdens (both in pancreas and liver) and CgA concentration $(P<0.001)$; changes in $\mathrm{CgA}$ and NSE concentrations also reflect treatment response $(P<0.001)$.

Conclusion: $\mathrm{CgA}$ and NSE are important diagnostic and follow-up markers in patients with NF-pNETs. The combined monitoring of CgA and NSE possesses more accuracy than individual values of $\mathrm{CgA}$ and NSE at predicting prognosis and disease progression.
\end{abstract}

\author{
Key Words \\ - chromogranin A \\ - neuron-specific enolase \\ - nonfunctioning pancreatic \\ neuroendocrine tumors \\ - prognosis prediction
}

\section{Introduction}

Pancreatic neuroendocrine tumors (pNETs) are regarded to originate from neuroendocrine cells in pancreas. pNETs can be classified as functioning, which are characterized by specific symptoms and relatively satisfying diagnostic rates owing to the hypersecretion of hormones or nonfunctioning based on the symptoms of space-occupying effects or the distant metastasis (1). Because of lack of specific manifestations, nonfunctioning pNETs (NF-pNETs) were more difficult to diagnose and follow-up.

Currently, for the NF-pNETs, imaging and pathological examination results still determine the suitable treatment decisions. However, traditional http://www.endocrineconnections.org https://doi.org/10.1530/EC-17-0276
(C) 2018 The authors Published by Bioscientifica Ltd

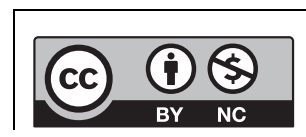

This work is licensed under a Creative Commons Attribution-NonCommercial 4.0 International License.
Endocrine Connections (2018) 7, 169-178 
imaging diagnosis techniques such as computed tomography (CT) and magnetic resonance imaging (MRI) have the comparatively lower level of sensitivity in the tumor diagnosis and progression detection and can hardly play the predictive role in the real-time monitoring for every patient; accurate pathological classification and stage of the surgical/needled specimen can give rise to the prognosis prediction and nonoperative management guidance to some extents, but this is usually based on a fixed small biopsy sample that may not reflect the heterogeneity present in advanced tumors (2). Additionally, as in other kinds of neoplasms, efforts are made to use selected circulating biomarker(s) to serve as the diagnosis forecaster, management instructions, monitoring of the efficacy of therapy and providers of the prognostic assessment (3). Thus, the identification of such a useful serological marker is in pressing need.

Chromogranin A (CgA), the first identified representative of granins, is produced and excreted by granules within both the classical endocrine glands and the diffuse neuroendocrine system. CgA is physiologically released by exocytosis and can be detected in blood. The expression of circulating CgA is closely correlated with the amount of secretory gland; thus once a tumor develops in an endocrine tissue, it becomes the main source of circulating $\mathrm{CgA}(4,5)$. Although $\mathrm{CgA}$ is recommended as the practical histopathological biomarkers by the latest guidelines of the consensus from European Neuroendocrine Tumors Society (ENETs) as well as the World Health Organization (WHO) $(6,7)$, the exploration of the clinical value of serum CgA for prediction of primary tumor load, therapeutic response and survival has not been sufficient in NF-pNET patients. In addition, controversies still remain on the diagnostic value (8), as well as the specific sensitivity and specificity of these measurable serum markers (9).

In this study, our aims were as follows: we recruited NF-pNETs, non-NETs neoplastic patients and healthy controls to investigate the clinical value of CgA assessment with respect to the neuron-specific enolase (NSE) in the diagnosis of NF-pNETs with the best cut-off value. Here, we present the clinical characteristics of NF-pNETs and the clinical value of serum CgA measurement in the evaluation of the relationship between the $\mathrm{CgA}$ concentration and those features; furthermore, we performed a combined test of CgA and NSE as a pair of oncological biomarkers to explore the value in NF-pNET patients, and then provide clinicians with useful evidence for the surveillance and management of patients with NF-pNETs.

$$
\text { http://www.endocrineconnections.org }
$$

\section{Materials and methods}

\section{Patients and control group}

This is a retrospective single-center clinical study performed at the Zhongshan Hospital, Shanghai, China. The study was approved by the Ethical Committee of Fudan University, and it followed the ethical guidelines of the Declaration of Helsinki. Related written informed consent was obtained from every recruited individual. Inclusion criteria of our research consisted of the availability of enhanced CT and MRI, and determination of CgA and NSE concentrations. Consecutive NF-pNET patients with pathologically confirmed diagnosis and controls were enrolled in our study. Participants were separately divided into several groups for clinical evaluation from our center from December 2011 to March 2016. The functional NET patients were excluded through the pathological results. Patients who had the past medical history of the increased aspartate transaminase, alanine transaminase, total bilirubin concentrations or the increased creatinine concentration caused by other diseases, drug treatment with proton pump inhibitors (PPIs) companied by less than 2 weeks of drug withdrawal and lack of histological confirmation were excluded. All the tests of serum CgA were performed when patients were not in the active stage of some specific inflammatory disease, such as systemic rheumatoid arthritis, systemic lupus erythematosus and COPD. Patients receiving long-term somatostatin analogs were permitted. To ensure the accuracy of the test and research, NF-pNET patients with the treatment history of other sources of tumors, namely no-NET neoplasms, were also eliminated from our study (even if they are very rare).

As for the control group, our study included 307 individuals: 99 healthy individuals (including 50 males and 49 females, range 19-82 years) and 208 patients (including 110 males and 98 females, range 17-87 years) (consisting of non-NET neoplastic patients, such as pancreatic ductal adenocarcinoma, rectal adenocarcinoma and benign gastroenteropancreatic tumors, etc.). All the individuals in the control group were selected following the principle of randomness.

In our study, all the cases and pathological sections were re-evaluated by another independent pathologist without the understanding of the tumor status according to the site of origin and criteria of the WHO and graded according to the ENETS proposal for grading and staging of NETs (10). Specifically, the 2010 WHO classification uses tumor-proliferative ability (including Ki-67 proliferation index and mitotic rate) to divide NETs into

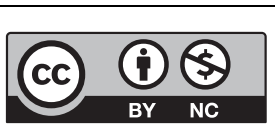

This work is licensed under a Creative Commons Attribution-NonCommercial 4.0 International License. 
three pathological grades: G1, G2 and G3. Of these, the G3 NF-pancreatic neuroendocrine carcinomas (NECs) are defined as a tumor with Ki-67 proliferation index greater than $20 \%$ or mitotic rate greater than 20 per 10 high power fields.

\section{The measurement of analyte}

Serum values of CgA were measured using an enzymelinked immunosorbent assay kit (IMRA, CisBio Bioassays Inc., France) according to protocol as the monoclonal antibodies in this kit capture human CgA proteins. The measurement range instructed by the manufacturer is between 7 and $870 \mu \mathrm{g} / \mathrm{L}$ and the recommended median normal value is $44 \mu \mathrm{g} / \mathrm{L}$. In our study, decreased or increased serum $\mathrm{CgA}$ concentrations were defined as a decrease or an increase by at least 50\% from the initial baseline; others were stable. The analytical coefficient of variation $(\mathrm{CV})$ of $\mathrm{CgA}$ is $148 \%$.

The concentration of serum NSE was measured by an electrochemiluminescence immunoassay automatic analyzer (Roche) and the recommended normal value is less than $15.2 \mu \mathrm{g} / \mathrm{L}$. The analytical CV of NSE is $92 \%$.

All the blood samples were collected in recruited individuals without any food intake or strenuous exercise to ensure standardization and precision of the results.

\section{Imaging quantification of the primary tumor size and liver metastatic tumor burden}

All the enrolled patients were subjected to multiphasic CT and MRI at the time of the initial evaluation. The image diagnostic reports were all assessed through a double-blinded manner; for outpatients, the preoperative radiographic data must be printed for evaluation and measurement. Routinely, the changes of all the patients in the post-treatment phase were evaluated from enhanced high-resolution (HR) CT with less than 5-mm slices. Therefore, additional HR-MRI can be performed under the condition that some obstructions can occur during the follow-up process.

\section{Treatment and follow-up}

All therapeutic protocols in our study group were implemented referring to the guideline of The National Comprehensive Cancer Network (NCCN) on Neuroendocrine Tumors (11). Selected patients underwent potentially curative resection of primary tumors in pancreas with lymph node dissection; other enrollees were subjected to a tumor biopsy. Comprehensive treatments were performed for patients with or without liver metastasis including liver-oriented strategies, such as hepatic segmental resection, hepatic trans-catheter arterial chemoembolization or radiofrequency ablation, and systemic therapy, which was octreotide long-acting release. Variances from imaging data in surgical area (location of primary tumor(s)) or liver metastatic tumor burden were defined as responses which include remission, partial response (PR), stable disease (SD), progressive disease (PD), progression, relapse and distant metastasis on the basis of response evaluation criteria in solid tumors (RECIST 1.1) (12) to assess the treatment response.

The data of preoperative clinical manifestations, radio-graphical information and blood biochemical examination, as well as the recovery processes were all recorded in all the NF-pNET patients. After the discharge, all the patients regularly followed up through the outpatient clinics or phone calls, as well as the common essential information of the tumor progression was also recorded accordingly. The follow-up period was as follows: the first follow-up was performed within 2-3 months after the baseline and the subsequent follow-up cycles usually ranged from 3 to 6 months or even shorter which depended on the clinical situations and(or) tumor relapse or metastasis being suspected. Data of overall survival (OS) and relapse/metastasis time were also documented. Duration of OS was calculated from the date of baseline to the last follow-up or tumor-specific death. The relapse time was computed from the date of complete tumor remission to recurrence.

\section{Statistical analysis}

Statistical analyses were performed using the SPSS statistical package (19.0; SPSS). CgA data of controls with NF-pNETs were used to construct the receiver operating characteristic (ROC) curve. The area under the ROC curve indicates the capability of biomarkers to discriminate between NET patients and controls; afterward, the sensitivity and specificity were calculated to determine the cut-off value. Pearson's $\chi^{2}$-test and Fisher's exact test were used to compare proportions when appropriate, whereas means were compared using a nonparametric test: the Mann-Whitney test or the Kruskal-Wallis test was used when there were more than two means. Pearson correlation and nonlinear exponential regression analysis were also carried out. 
OS analyses were carried out using the Kaplan-Meier method and the results were compared using a logrank test. A multivariable Cox proportional hazards model predicting OS was used using backward stepwise selection. Risk factors were expressed as the hazard ratio (HR, 95\% confidence interval (CI)). Statistical significance was defined as $P$ value less than 0.05 .

\section{Results}

\section{Clinical characteristics of recruited people}

From December 2011 to March 2016, a total of 474 individuals (two patients were excluded because of the late-stage renal failure and accompanied with the history of prostate cancer separately) were enrolled in our study, including 167 NF-pNET patients, 208 non-NET neoplastic patients and 99 healthy individuals. Table 1 summarizes the patient characteristics, biomarker concentration, tumor grade, CgA values and NSE values. The diagnoses of 167 NF-pNET patients were re-confirmed by pathologists according to WHO grade, and the numbers of grades G1, G2 and G3 were 36 (21.6\%), 102 (61.1\%) and 29 (17.4\%), respectively. There was a significant difference between NF-pNETs and control groups on serum CgA concentration $(P=0.001)$. The mean CgA values of different groups were 108,78 and $42 \mu \mathrm{g} / \mathrm{L}$.
The correlation between the diagnosis and the $\mathrm{CgA}$ concentration in NF-pNETs

In order to identify the diagnostic value of serum $\mathrm{CgA}$ for distinguishing NF-pNETs from controls, the ROC curve and analysis were constructed by our CgA values in 167 NF-pNET patients and 99 healthy controls. The most optimal cut-off value for NF-pNETs indicated by our data was $62 \mu \mathrm{g} / \mathrm{L}$, with $68.3 \%$ sensitivity and $85.6 \%$ specificity, and the Area Under Curve (AUC) was 0.83; in addition, with respect to the NSE on NF-pNET patients without remission, the best threshold was $13 \mu \mathrm{g} / \mathrm{L}$, resulting in a specificity of $63.7 \%$ and a sensitivity of $74.8 \%$, with AUC index 0.71. Furthermore, when the two biomarkers were combined using the binary logistic modeling, AUC was significantly increased to 0.90 (Fig. 1), which demonstrated that the elevated CgA and NSE concentrations provide a relatively high possibility of NET disease.

In our study, the CgA concentrations were statistically correlated with the tumor grade in well-differentiated NF-pNETs, demonstrating that the mean CgA indexes of G1 and G2 NF-pNETs were, respectively, $78 \mu \mathrm{g} / \mathrm{L}$ and $153 \mu \mathrm{g} / \mathrm{L}(P=0.02)$; however, they were not statistically significant between well-differentiated pNETs and poorly-differentiated pNETs $(P=0.09)$. For NSE, the mean values of G1, G2 and G3 PNETs were 13, 17 and $21 \mu \mathrm{g} / \mathrm{L}$ $(P=0.008)$, respectively, showing statistical significance on implications for diverse grades, as shown in Fig. 2 and

Table 1 Common characteristics of patients and controls.

\begin{tabular}{|c|c|c|c|c|c|c|c|}
\hline \multirow[b]{3}{*}{ Characteristics } & \multirow[b]{2}{*}{ NF-pNETs } & & \multicolumn{4}{|c|}{ Groups } & \multirow[b]{3}{*}{ P-Value } \\
\hline & & & Non-NETs & & Healthy & & \\
\hline & No. & $\%$ & No. & $\%$ & No. & $\%$ & \\
\hline No. of individuals & 167 & & 208 & & 99 & & \\
\hline Mean age, years & 50.0 & & 58.1 & & 44.4 & & 0.091 \\
\hline S.D. & 17.4 & & 17.0 & & 22.5 & & \\
\hline \multicolumn{8}{|l|}{ Gender distribution } \\
\hline Males & 82 & 49 & 110 & 53 & 50 & 51 & 0.170 \\
\hline Females & 87 & 51 & 98 & 47 & 49 & 49 & \\
\hline LM & 47 & 28 & & & & & \\
\hline $\mathrm{CgA}(\mu \mathrm{g} / \mathrm{L})$ & & & & & & & $0.000 *$ \\
\hline Median & 67.0 & & 62.3 & & 58.7 & & \\
\hline Mean & 107.9 & & 78.1 & & 42.2 & & \\
\hline S.D. & 136.0 & & 67.3 & & 32.5 & & \\
\hline NSE $(\mu \mathrm{g} / \mathrm{L})$ & & & & & & & $0.003 *$ \\
\hline Median & 13.6 & & 11.2 & & & & \\
\hline Mean & 15.2 & & 12.6 & & & & \\
\hline S.D. & 12.2 & & 14.6 & & & & \\
\hline Tumor grade & & & & & & & 0.000 \\
\hline G1 & 36 & 22 & & & & & \\
\hline $\mathrm{G} 2$ & 102 & 61 & & & & & \\
\hline G3 & 29 & 17 & & & & & \\
\hline
\end{tabular}

CgA, chromogranin A; LM, liver metastasis; non-NETs, non-NETs neoplastic patients; NSE, neuron-specific enolase.

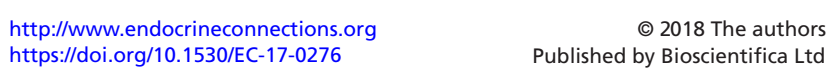

This work is licensed under a Creative Commons Attribution-NonCommercial 4.0 International License. 
ROC curve of NSE on PNETs
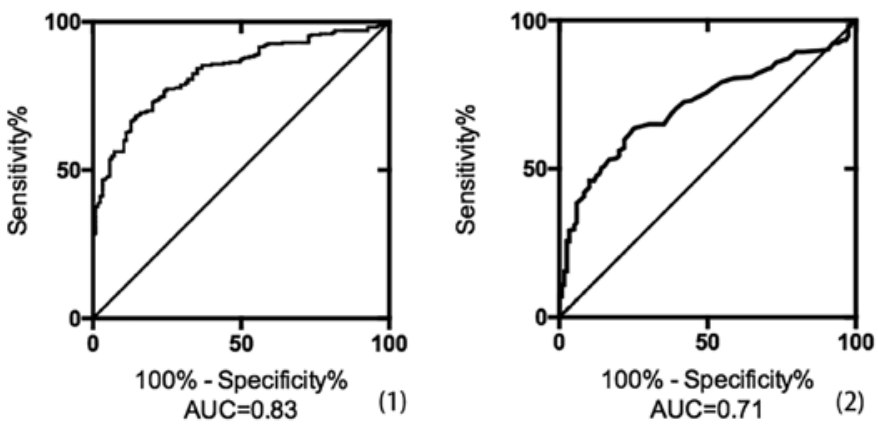

\section{Figure 1}

Receiver operating characteristic (ROC) curves separately demonstrating the diagnostic value of chromogranin A (CgA), neuron-specific enolase (NSE) and the combined markers in NF-pNETs; the areas under the curve (AUCs) were separately $0.83,0.71$ and 0.90 ; (1) the most optimal cut-off value of CgA for NF-pNETs indicated by our data was $61.8 \mu \mathrm{g} / \mathrm{L}$, with $68.3 \%$ sensitivity and $85.6 \%$ specificity; (2) the best cut-off value of NSE for NF-pNETs was $13.2 \mu \mathrm{g} / \mathrm{L}$, with $63.7 \%$ sensitivity and $74.8 \%$ specificity; (3) the binary logistic regression modeling was used to construct the curve; the AUCs were significantly increased.

Table 2. Besides, on the aspects of pNETs, at its optimal cut-off for the prediction of LM determined by ROC curve analyses (in Fig. 3 and Table 3), the combination of CgA and NSE differentiated pNET and pNEC with LM from nonmetastatic NET disease with very high SNs/SPs of 78.3\%/66.8\%, 88.9\%/70.8\%, respectively.

\section{Indicative value of $\mathrm{CgA}$ concentration on primary tumor size and on liver metastasis tumor load}

In the present study, the tumor size of NF-pNETs on the pre-treatment period was also recorded. For the enrolled outpatients, the preoperative radiographic data must be printed for evaluation and measurement. Of the 167 NF-pNETs, $40.7 \%$ of the origin site was pancreatic head $(n=68), 52.1 \%$ was the distal pancreas $(n=87)$ and $7.19 \%$ was the neck and body of pancreas $(n=12)$. The median tumor diameter was $2.90 \pm 1.81 \mathrm{~cm}$, with the average diameters of males and females being
$3.00 \pm 1.63 \mathrm{~cm}$ and $2.50 \pm 1.96 \mathrm{~cm}(P=0.66)$, respectively. To determine the relationship between serum biomarkers and tumor sizes in NF-pNETs, the scatter diagrams of tumor diameter and CgA (and NSE) distribution were drawn using the regression analysis (Fig. 4), showing that there was a nonlinear exponential regression between primary tumor diameters $(X)$ and $\mathrm{CgA}(Y)$ concentration $\left(Y=18.56^{\star} X+39.40, F=9.73, R=0.79, P=0.002\right)$. However, same statistical correlation did not exist for NSE values $\left(Y=-0.2465^{*} X+15.06, \quad F=0.19, P=0.67\right)$. Beyond that, when primary tumor diameter was regarded as less than $1 \mathrm{~cm}, 1-3 \mathrm{~cm}$ or greater than $3 \mathrm{~cm}$, according to the scatterplot, the CgA concentration was correlated with the degree of tumor load extension $(P<0.001)$.

In our NF-pNET patient group, all patients with metastatic disease presented with liver metastases, except for one patient who had the spinal bone metastases only (excluded already). 28.1\% of the NF-pNET patients have shown simultaneous or heterogeneous LM.
A The column of the mean $\mathrm{CgA}$ with $95 \% \mathrm{Cl}$ for varied Grade

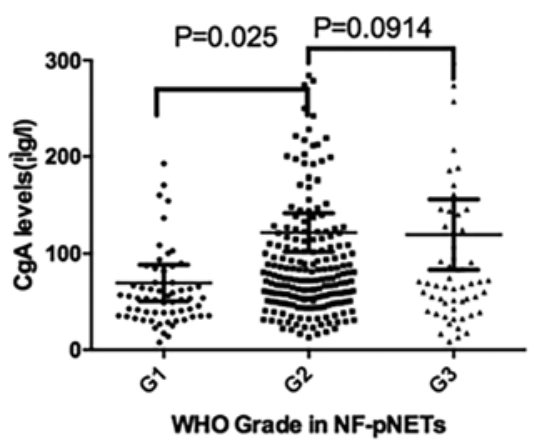

B The column of the mean NSE with $95 \% \mathrm{Cl}$ for varied Grade

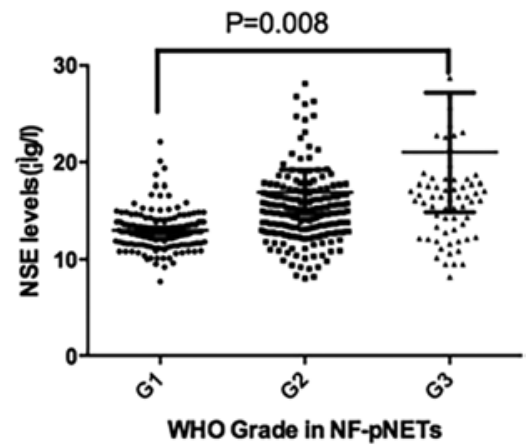

Figure 2

CgA concentration (A) and NSE concentration (B) in relation to the WHO tumor grade of NF-PNETS. $\mathrm{CgA}$, chromogranin A; NSE, neuron-specific enolase. http://www.endocrineconnections.org https://doi.org/10.1530/EC-17-0276 (c) 2018 The authors Published by Bioscientifica Ltd

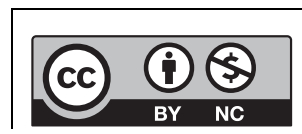

This work is licensed under a Creative Commons Attribution-NonCommercial 4.0 International License. 
Table $2 \mathrm{CgA}$ and NSE values vary in the different tumor grades of 167 NF-pNETs.

\begin{tabular}{|c|c|c|c|c|c|}
\hline & $\boldsymbol{N}$ & Mean & Median & Range & P-Value \\
\hline \multicolumn{6}{|l|}{ CgA levels ( $\mu \mathrm{g} / \mathrm{L})$} \\
\hline Tumor grade & & & & & 0.027 \\
\hline G1 & 36 & 78.1 & 56.2 & $7.0-468.2$ & \\
\hline G2 & 102 & 153.3 & 74.3 & $7.7-839.5$ & \\
\hline G3 & 29 & 191.1 & 74.2 & $36.3-870.0$ & \\
\hline \multicolumn{6}{|l|}{ NSE levels $(\mu \mathrm{g} / \mathrm{L})$} \\
\hline Tumor grade & & & & & 0.008 \\
\hline G1 & 36 & 13.0 & 12.9 & $7.7-22.1$ & \\
\hline G2 & 102 & 17.0 & 14.9 & $8.0-204.7$ & \\
\hline G3 & 29 & 21.0 & 16.5 & $8.2-193.3$ & \\
\hline
\end{tabular}

CgA, chromogranin A; NSE, neuron-specific enolase.

For recruited NF-pNET patients, the mean CgA value of patients with and without LM was, respectively, $230 \pm 22 \mu \mathrm{g} / \mathrm{L}$ and $84 \pm 6 \mu \mathrm{g} / \mathrm{L}$ ( $R$ square: $0.14, P<0.0001)$. Meanwhile, the serum NSE concentration showed a similar statistical trend on LM from regression analysis (mean values: $20 \pm 2 \mu \mathrm{g} / \mathrm{L}$ vs $14 \pm 0.2 \mu \mathrm{g} / \mathrm{L}, \quad R$ square: $0.04, P<0.0001$ ) (Fig. 5, $(1,2)$ ). In addition, scatterplots based on linear regression were manufactured for the relations between metastatic liver tumor burden $(X)$ and $\mathrm{CgA}(Y)$ concentration $\left(Y=0.8823^{*} X+119.6\right.$, $F=53.93, \quad R=0.2932, \quad P<0.001)$; with respect to NSE concentration, no differences were found from the analysis $(Y=0.007240 * X+19.24, F=0.25, R=0.002062$, $P=0.61$ ) (Fig. 5, $(3,4)$ ). Further to the observation, the elevated serum CgA concentrations have a stronger influence on hepatic tumor load spread compared with NSE concentration if both biomarkers were evaluated simultaneously. On the follow-up process, we obtained additional evidence for the assumption that a sudden and rapid increase in serum $\mathrm{CgA}$ in patients with metastatic disease (included in the PD group) serves as an indicator of tumor progression.

\section{$\mathrm{CgA}$ and NSE as a pair of predictive biomarkers on therapeutic response and survival in NF-pNET patients}

The median follow-up time was 55.5 months. Concerning the therapeutic response of NF-pNETs, patients defined as demonstrating a PR and a complete response (namely remission) $(n=133)$ during the treatment process had decreased CgA concentration, whereas patients regarded as having $\mathrm{PD} /$ relapse $(n=83)$ had increased $\mathrm{CgA}$ concentration, (Fisher's exact test, $P<0.001$ ) (Table 4 ). The results of the univariate analysis are listed in Table 5. Multivariate analyses demonstrated that serum CgA, primary tumor diameter and age were independent risk factors for tumor recurrence and OS.

To investigate the prognostic value for OS of $\mathrm{CgA}$ and NSE, Cox regression analyses were performed. Figure 6 summarizes the comparison of OS in different marker concentrations in well-differentiated NF-pNET patients and NF-pNEC patients, respectively. The median survival time for all the 167 recruited NF-pNET patients was $50.3 \pm 6.3$ months. From our study, well-differentiated NF-pNET patients of the elevated CgA concentration subsection showed a significantly lower OS than those in the normal $\mathrm{CgA}$ concentration subgroup or decreased CgA concentration subgroup $(P<0.0001$; mean OS: 29.3, 50.3 and 51.2 months, respectively). Among them, there was no significant difference of the OS between the normal CgA concentration subgroup and the decreased CgA concentration subgroup $(P=0.06)$. Besides this, the concentration of NSE also showed significance of OS in well-differentiated NF-pNET patients $(P=0.007$; mean OS: 26.3 months and 44.6 months). However, for NF-pNEC patients, our data showed no significance of OS between the elevated CgA concentration subgroup and the normal/ decreased subgroups.
A

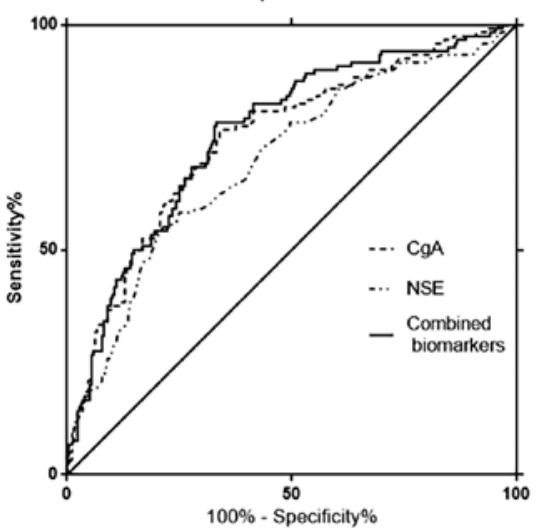

B

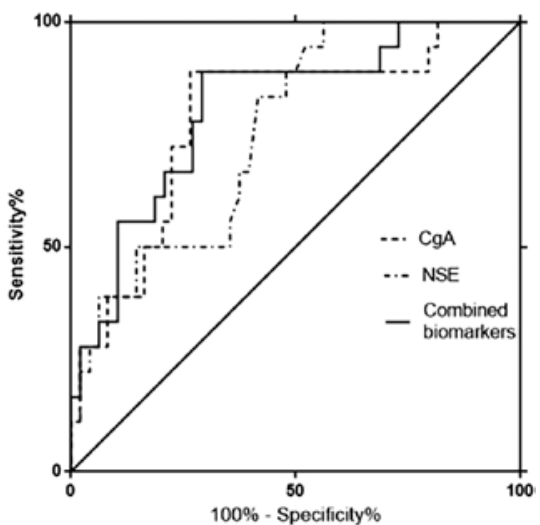

Figure 3

Receiver operating characteristic (ROC) curves separately demonstrating the value of chromogranin A ( $\mathrm{CgA})$, neuron-specific enolase (NSE) for the prediction of LM in NF-pNETs (A) and NF-p-NECs (B); specific areas under the curve of each biomarker are enumerated in Table 3; $P$-value $\leq 0.0001$ http://www.endocrineconnections.org https://doi.org/10.1530/EC-17-0276
() 2018 The authors Published by Bioscientifica Ltd
This work is licensed under a Creative Commons Attribution-NonCommercial 4.0 International License. 
Table 3 Diagnostic performance of CgA, NSE and combined biomarkers on NF-pNETs for LM.

\begin{tabular}{|c|c|c|c|c|c|c|}
\hline & Sensitivity (\%) & Specificity (\%) & Cut-off value $(\mu \mathrm{g} / \mathrm{L})$ & AUC & PPV $(\%)$ & NPV $(\%)$ \\
\hline \multicolumn{7}{|l|}{$\mathrm{CgA}$} \\
\hline PNET with LM vs with no LM & 78.3 & 65.5 & 74.0 & 0.753 & 51.5 & 86.6 \\
\hline $\begin{array}{l}\text { PNEC with LM vs with no LM } \\
\text { NSE }\end{array}$ & 55.7 & 78.3 & 15.0 & 0.785 & 54.6 & 79.1 \\
\hline PNET with LM vs with no LM & 93.8 & 72.9 & 58.5 & 0.704 & 66.7 & 95.3 \\
\hline $\begin{array}{l}\text { PNEC with LM vs with no LM } \\
\text { Combined biomarkers }\end{array}$ & 87.5 & 58.3 & 16.4 & 0.757 & 54.9 & 89.0 \\
\hline PNET with LM vs with no LM & 78.3 & 66.8 & & 0.758 & 56.5 & 86.8 \\
\hline PNEC with LM vs with no LM & 88.9 & 70.8 & & 0.808 & 61.8 & 91.7 \\
\hline
\end{tabular}

CgA, chromogranin A; LM, liver metastasis; NPV, negative predictive value; NSE, neuron-specific enolase; pNETs, Pancreatic neuroendocrine tumors; PPV, positive predictive value.

For the combined monitoring of $\mathrm{CgA}$ and NSE for all the NF-pNET patients, the elevated expression of both serum CgA and NSE showed a shorter OS than monotonously elevated or sustained biomarkers in well-differentiated NF-pNET patients $(P<0.001$; OS: 23.2, 38.6 and 49.6 months, respectively). Nevertheless, same statistical difference did not appear in NF-pNEC patients $(P=0.13)$.

\section{Discussion}

Since the concept of NETs is familiar (13) and the patients have to visit the surgeons, a biomarker which reflects the status of $\mathrm{CgA}$ test is desirable. Some studies concerning the clinical role of $\mathrm{CgA}$ were identified but the volume of the exploration for the diagnostic value of $\mathrm{CgA}$ was low $(9,14,15)$. Meanwhile, majority of the research included a relatively small size of samples because NETs are a kind of rare disease. Our study demonstrated in accordance with the previous reports (16) that serum CgA is an effective diagnostic biomarker in NF-pNET patients and the measurement of circulating $\operatorname{CgA}$ can be used as a specific diagnostic tool for the prediction of NETs in different treatment periods. Therefore, because of the heterogeneity of the NETs, the anatomically wide distribution of the endocrine system in human beings and the lack of standardized test methods and research design, it is difficult to determine the role of CgA in NETs with previous studies. In our research, 474 individuals with informed consent from either clinics or surgical wards were recruited (containing a quite volume of people in both research cohort and control cohort). In addition, evaluation of the primary/metastatic tumor burden and the therapeutic response as well as the treatment choices were performed based on international standards (11). In order to avoid the objective bias on the scientificity, utility and veracity in monitoring and assessing the CgA concentration (such as PPI drugs and food intake, baseline renal failure, etc.) of NF-pNETs and controls, a series of strict inclusion and exclusion criteria were formulated for the study. Furthermore, both the pathological type and the test index were re-confirmed by another independent and professional doctor from related medical departments.

\section{A correlation of $\mathrm{CgA}$ levels with primary} tumor diameter

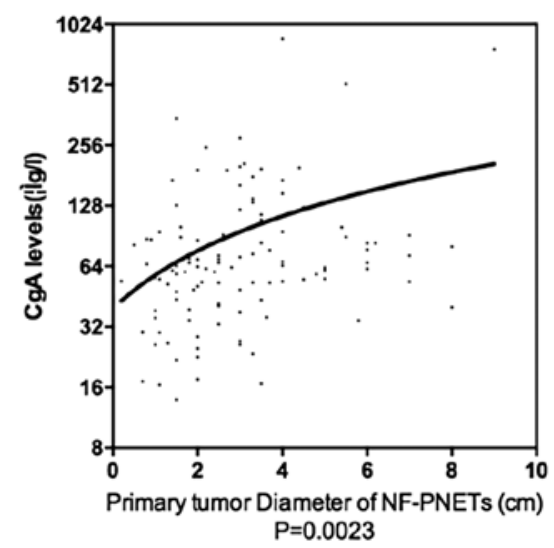

B correlation of NSE levels with Primary tumor Diameter

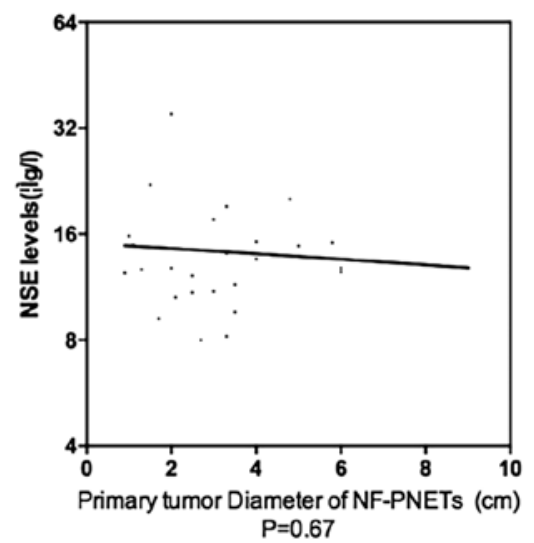

Figure 4

The scatter diagram demonstrating the correlation of NF-pNET tumor diameter with CgA concentration $(P=0.0023)(A)$ and NSE concentration $(P=0.67)(B)$ accompanied by the $Y$-axis on $\log 2$ scale. The function formula was $Y=18.56^{*} X+39.40$ and $Y=-0.2465^{*} X+15.06$, separately. CgA, chromogranin A; NSE, neuronspecific enolase.
@) 2018 The authors
Published by Bioscientifica Ltd http://www.endocrineconnections.org https://doi.org/10.1530/EC-17-0276

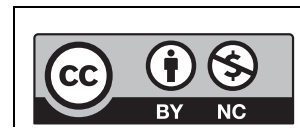

This work is licensed under a Creative Commons Attribution-NonCommercial 4.0 International License. 


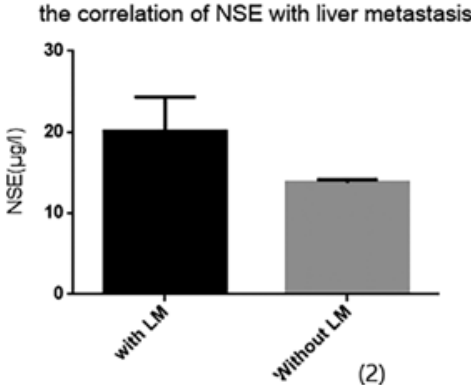

NF-NETs with and without liver metastasis

NF-NETs with and without liver metastasis

correlation of $\mathrm{CgA}$ levels with liver metastasis load

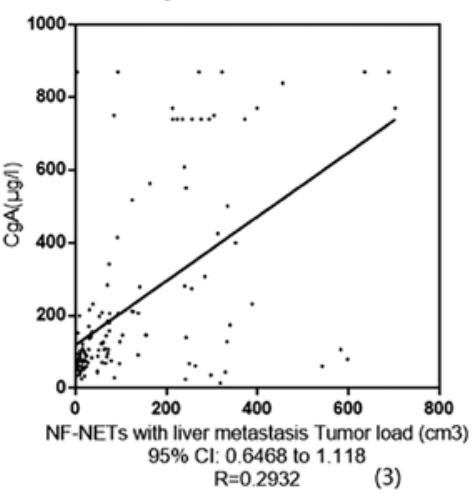

correlation of NSE levels with liver metastasis load

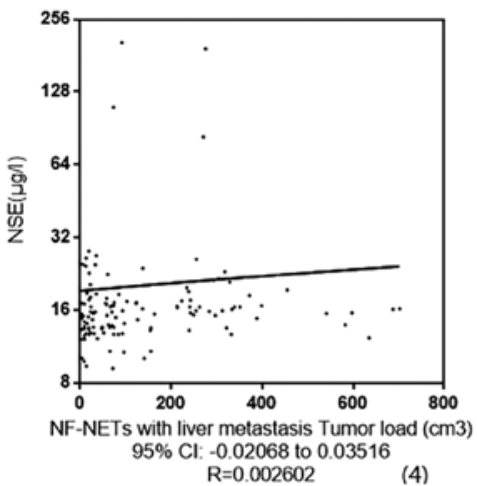

\section{Figure 5}

The correlation between liver metastasis and our biomarker concentrations in the NF-pNET group: (1) $\mathrm{CgA}$ concentration was related to the LM of NF-pNETs $(P<0.0001)$. (2) NSE concentration was correlated with LM $(P<0.0001)$. (3) The CgA concentration correlated with liver metastatic tumor burden and there was a linear regression between them. (4) The NSE concentration correlated with liver metastatic tumor burden. $\mathrm{CgA}$, chromogranin A; NF-pNETs, nonfunctional gastroenteropancreatic neuroendocrine tumors; NSE, neuron-specific enolase.
Here, we provide additional evidence that CgA concentrations are predictive for tumor activity. CgA can be regarded as an early biomarker of NF-pNETs, which was significantly correlated with the tumor grade, and presented to show better pertinence than NSE in wellmoderate NF-pNETs; however, our data indicated that the CgA and NSE values of distinguishing NETs from NECs were not significantly valid $(P=0.09)$. As indicated by our data, the corresponding sensitivity and specificity were $68.3 \%$ and $85.6 \%$ in NF-pNETs, which are generally consistent with the previous reports (9). What is more, the

Table $4 \mathrm{CgA}$ and NSE values vary in the different grades of NF-pNETs.

\begin{tabular}{|c|c|c|c|c|c|}
\hline & $\boldsymbol{N}$ & Mean & Median & Range & P-Value \\
\hline \multicolumn{6}{|c|}{ CgA level ( $\mu \mathrm{g} / \mathrm{L})$} \\
\hline Status of test & & & & & 0.012 \\
\hline PR & 53 & 127.3 & 72.8 & $5.3-501.1$ & \\
\hline PD & 83 & 272.7 & 124.9 & $37.2-870.0$ & \\
\hline Remission & 53 & 47.0 & 45.7 & $5.6-132.8$ & \\
\hline SD & 44 & 103.1 & 74.3 & $5.4-740.0$ & \\
\hline \multicolumn{6}{|c|}{ NSE level $(\mu \mathrm{g} / \mathrm{L})$} \\
\hline \multicolumn{6}{|c|}{ Status of test } \\
\hline PR & 53 & 16.8 & 16.0 & $9.6-31.0$ & 0.022 \\
\hline PD & 83 & 27.9 & 15.7 & $9.2-204.7$ & \\
\hline Remission & 53 & 12.9 & 12.5 & $7.7-26.8$ & \\
\hline SD & 44 & 14.8 & 13.8 & $9.0-26.3$ & \\
\hline
\end{tabular}

$\mathrm{CgA}$, chromogranin A; NSE, neuron-specific enolase; PD, progressive disease; PR, partial response; SD, stable disease.

http://www.endocrineconnections.org

https://doi.org/10.1530/EC-17-0276

(c) 2018 The authors Published by Bioscientifica Ltd
AUC has dramatically increased when the circulating NSE biomarkers were added to the test with $\mathrm{CgA}$, which shows a promising trend for the diagnosis of NF-pNETs. On primary tumor load in NF-pNETs, our study preliminarily indicated that there was a correlation between primary tumor diameters and $\mathrm{CgA}$ concentration, while increased NSE concentration did not showed the significance.

Tumor recurrence and metastasis were widely recognized as the most essential factors regarding the survival and prognosis of patients. Our study had a more practical approach for the pNETs at present. Comparing the CgA concentration from the LM subgroup and no LM subgroup by linear regression, CgA was significantly associated with LM tumor burden and showed the relation to the extent of liver metastatic spread. Data on CgA response of diverse status from this study are someway different from those of Kos (17), who found a significant CgA decrease in only the subgroup of NET

Table 5 Multivariable Cox proportional model of NF-pNET cohort.

\begin{tabular}{|c|c|c|c|}
\hline Variables & $\boldsymbol{P}$ & HR & $95 \% \mathrm{Cl}$ \\
\hline Age & 0.000 & 2.2 & $1.2-3.8$ \\
\hline CgA levels & 0.001 & 6.2 & $1.9-20.2$ \\
\hline Baseline primary tumor load & 0.045 & 1.18 & $0.99-1.36$ \\
\hline
\end{tabular}

$\mathrm{CgA}$, chromogranin $\mathrm{A} ; \mathrm{Cl}$, confidence interval; $\mathrm{HR}$, hazard ratio.

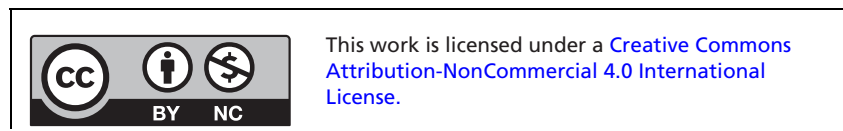


A The comparison of OS between different CgA levels

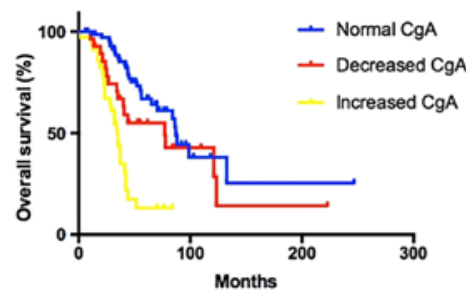

$C^{\text {The comparison of OS between different NSE levels }}$

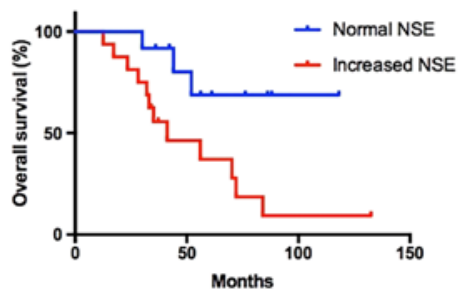

B The comparison of OS between different NSE levels

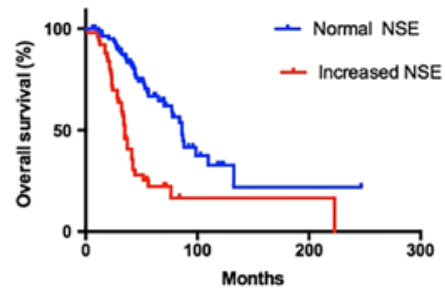

D

OS between different biomakers levels

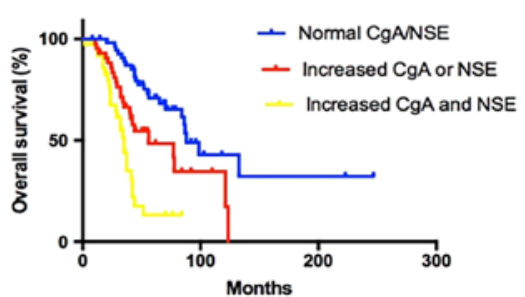

Figure 6

Univariate analysis showed the prognostic value of CgA and NSE for NF-pNETs. (A) and (B) The comparison of OS between different NSE or CgA concentrations from the initial baseline in well-differentiated NF-pNET patients. (C) The comparison of OS between different NSE concentrations from the initial baseline in NF-pNEC patients. (D) The comparison of OS between different biomarker concentrations in NF-pNET patients. CgA, chromogranin A; NSE, neuron-specific enolase; NF-pNETs, nonfunctional pancreatic neuroendocrine tumors; OS, overall survival. patients with high basal CgA concentration. We found that the $\mathrm{CgA}$ concentration of complete response to treatment demonstrated a downward trend and had no difference compared with the control cohort $(P<0.0001)$. Moreover, it must be noted that their subgroup of PR NET patients with slightly elevated basal CgA concentration was heterogeneous with a previous radical surgery in other hospitals accompanied by unclear medical history statement in some cases. It is obvious that the PD cohort patients showed higher CgA and NSE concentrations $(P<0.001)$, in accord with the conclusion that a $>30 \%$ increase in $\mathrm{CgA}$ concentration after acute systematic therapeutic administration has a relevant prognostic value as previously reported (18).

The molecular mechanisms of analyte for NETs were widely explored in recent years, as an on/off switch and $\mathrm{CgA}$ which control the dense-core secretory granule biogenesis in endocrine cells. The synthesis of CgA itself can be regulated by hormones and can serve as a guide in controlling hormone secretion (8). Studies have also reported that the combination of $\mathrm{CgA}$ and other serum biomarkers can be recommended in patients with welldifferentiated NETs (5). The clinical values of serum CgA and NSE were explored from our data. Moreover, a preliminary integrated monitoring of serum CgA and NSE was conducted in this study. In our study, of the prognostic parameters for OS, CgA was the most significant, and the combined detection of CgA and NSE was a more accurate prognostic variable for well-differentiated NF-pNET patients. Kaplan-Meier curves clearly indicate an association between CgA and NSE concentrations and survival (Fig. 6). Considering a majority proportion of WHO G1 and G2 NF-pNET patients, it is clear that within all histological NF-pNET groups, a combination of multiple biomarkers proved to be more sensitive for identifying and discriminating the disease than each marker alone.

However, there are still some possible limitations for the combined monitoring: the first factor is related to monotonous category of NF-pNETs; the clinical values of such a pair of serum oncological markers in NETs from gastrointestinal or other systems were not explored. The anatomical complexity with varied range of histology and the diversity of treatment options in gastrointestinal tract make the research difficult to perform. Furthermore, more detailed research works of the tumor indicators for prediction of NETs in specific organs are still required in the future.

In summary, we found that the sensitivity and specificity of $\mathrm{CgA}$ are optimal and are good biomarkers in patients with NF-pNETs; NSE enhances the diagnostic value of CgA for NF-pNETs. We also proved the value of combined test of $\mathrm{CgA}$ and NSE in reflecting primary and metastatic tumor burden(s), indicating therapeutic response during treatment. Furthermore, the combined test of CgA and NSE revealed a more certain and accurate significance correlated with clinical characteristics and, particularly, the prognosis.

Declaration of interest

The authors declare that there is no conflict of interest that could be perceived as prejudicing the impartiality of the research reported.

\section{Funding}

This study was supported by National Natural Science Foundation of China (81401923 and 81572294), CSCO-Novartis neuroendocrine tumor development fund (2013) and Novartis China GEP/NET Registry (CSMS955ACN11).

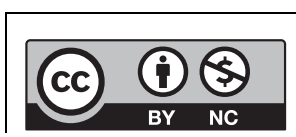

This work is licensed under a Creative Commons Attribution-NonCommercial 4.0 International License. 


\section{Acknowledgements}

The authors thank all the doctors and nurses during the diagnosis, treatment and the detection process.

\section{References}

1 Pavel M, O’Toole D, Costa F, Capdevila J, Gross D, Kianmanesh R, Krenning E, Knigge U, Salazar R, Pape UF, et al. ENETS consensus guidelines update for the management of distant metastatic disease of intestinal, pancreatic, bronchial Neuroendocrine Neoplasms (NEN) and NEN of unknown primary site. Neuroendocrinology 2016 103 172-185. (https://doi.org/10.1159/000443167)

2 Gerlinger M, Rowan AJ, Horswell S, Larkin J, Endesfelder D, Gronroos E, Martinez P, Matthews N, Stewart A, Tarpey P, et al. Intratumor heterogeneity and branched evolution revealed by multiregion sequencing. New England Journal of Medicine 2012366 883-892. (https://doi.org/10.1056/NEJMoa1113205)

3 Gut P, Czarnywojtek A, Fischbach J, Baczyk M, Ziemnicka K, Wrotkowska E, Gryczynska M \& Ruchala M. Chromogranin A unspecific neuroendocrine marker. Clinical utility and potential diagnostic pitfalls. Archives of Medical Science 201612 1-9. (https://doi.org/10.5114/aoms.2016.57577)

4 Kim T, Tao-Cheng JH, Eiden LE \& Loh YP. Chromogranin A, an 'on/off' switch controlling dense-core secretory granule biogenesis. Cell 2001106 499-509. (https://doi.org/10.1016/ S0092-8674(01)00459-7)

5 Korse CM, Taal BG, de Groot CA, Bakker RH \& Bonfrer JM. Chromogranin-A and N-terminal pro-brain natriuretic peptide: an excellent pair of biomarkers for diagnostics in patients with neuroendocrine tumor. Journal of Clinical Oncology 200927 4293-4299. (https://doi.org/10.1200/JCO.2008.18.7047)

6 Luo G, Javed A, Strosberg JR, Jin K, Zhang Y, Liu C, Xu J, Soares K, Weiss MJ, Zheng L,et al. Modified staging classification for pancreatic neuroendocrine tumors on the basis of the American Joint Committee on Cancer and European Neuroendocrine Tumor Society Systems. Journal of Clinical Oncology 201735 274-280. (https://doi. org/10.1200/JCO.2016.67.8193)

7 Reid MD, Balci S, Saka B \& Adsay NV. Neuroendocrine tumors of the pancreas: current concepts and controversies. Endocrine Pathology 201425 65-79. (https://doi.org/10.1007/s12022-013-9295-2)

8 Paik WH, Ryu JK, Song BJ, Kim J, Park JK, Kim YT \& Yoon YB. Clinical usefulness of chromogranin a in pancreatic neuroendocrine neoplasm. Journal of Korean Medical Science 201328 750-754. (https://doi.org/10.3346/jkms.2013.28.5.750)

9 Modlin IM, Gustafsson BI, Moss SF, Pavel M, Tsolakis AV \& Kidd M. Chromogranin A - biological function and clinical utility in neuro endocrine tumor disease. Annals of Surgical Oncology 201017 2427-2443. (https://doi.org/10.1245/s10434-010-1006-3)

10 Halfdanarson TR, Rabe KG, Rubin J \& Petersen GM. Pancreatic neuroendocrine tumors (PNETs): incidence, prognosis and recent trend toward improved survival. Annals of Oncology 200819 1727-1733. (https://doi.org/10.1093/annonc/mdn351)

11 Kulke MH, Shah MH, Benson AB 3rd, Bergsland E, Berlin JD, Blaszkowsky LS, Emerson L, Engstrom PF, Fanta P, Giordano T, et al. Neuroendocrine tumors, version 1.2015. Journal of the National Comprehensive Cancer Network 201513 78-108. (https://doi. org/10.6004/jnccn.2015.0011)

12 Eisenhauer EA, Therasse P, Bogaerts J, Schwartz LH, Sargent D, Ford R, Dancey J, Arbuck S, Gwyther S, Mooney M, et al. New response evaluation criteria in solid tumours: revised RECIST guideline (version 1.1). European Journal of Cancer 200945 228-247. (https://doi.org/10.1016/j.ejca.2008.10.026)

13 Yao JC, Hassan M, Phan A, Dagohoy C, Leary C, Mares JE, Abdalla EK, Fleming JB, Vauthey JN, Rashid A, et al. One hundred years after "carcinoid": epidemiology of and prognostic factors for neuroendocrine tumors in 35,825 cases in the United States. Journal of Clinical Oncology 200826 3063-3072. (https://doi.org/10.1200/ JCO.2007.15.4377)

14 Chou WC, Hung YS, Hsu JT, Chen JS, Lu CH, Hwang TL, Rau KM, Yeh KY, Chen TC \& Sun CF. Chromogranin A is a reliable biomarker for gastroenteropancreatic neuroendocrine tumors in an Asian population of patients. Neuroendocrinology 201295 344-350. (https://doi.org/10.1159/000333853)

15 Singh S \& Law C. Chromogranin A: a sensitive biomarker for the detection and post-treatment monitoring of gastroenteropancreatic neuroendocrine tumors. Expert Review of Gastroenterology and Hepatology 20126 313-334. (https://doi.org/10.1586/egh.12.15)

16 Han X, Zhang CY, Tang M, Xu XF, Liu LX, Ji Y, Pan BS \& Lou WH. The value of serum chromogranin $A$ as a predictor of tumor burden, therapeutic response, and nomogram-based survival in well-moderate nonfunctional pancreatic neuroendocrine tumors with liver metastases. European Journal of Gastroenterology and Hepatology 201527 527-535. (https://doi.org/10.1097/ MEG.0000000000000332)

17 Karavitaki N, Botusan I, Radian S, Coculescu M, Turner HE \& Wass JAH. The value of an acute octreotide suppression test in predicting long-term responses to depot somatostatin analogues in patients with active acromegaly. Clinical Endocrinology 200562 282-288. (https://doi.org/10.1111/j.1365-2265.2004.02191.x)

18 Massironi S, Conte D, Sciola V, Spampatti MP, Ciafardini C, Valenti L, Rossi RE \& Peracchi M. Plasma chromogranin A response to octreotide test: prognostic value for clinical outcome in endocrine digestive tumors. American Journal of Gastroenterology $2010 \mathbf{1 0 5}$ 2072-2078. (https://doi.org/10.1038/ajg.2010.154)

Received in final form 9 November 2017

Accepted 30 November 2017

Accepted Preprint published online 30 November 2017 http://www.endocrineconnections.org https://doi.org/10.1530/EC-17-0276
(C) 2018 The authors Published by Bioscientifica Ltd

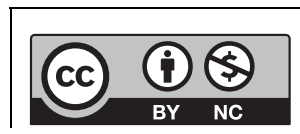

This work is licensed under a Creative Commons Attribution-NonCommercial 4.0 International License. 